\title{
AVALIAÇÃO DO COMPORTAMENTO TÉRMICO DA REAÇÃO DE REFORMA AUTOTÉRMICA DO METANO UTILIZANDO MAXIMIZAÇÃO DE ENTROPIA
}

\author{
R.H.N.FRAZÃO ${ }^{1}$, A.D.S. VIDOTTI ${ }^{1}$, R. GUIRARDELLO ${ }^{2}$, A.C.D.FREITAS ${ }^{1}$
}

${ }^{1}$ Universidade Federal do Maranhão, Coordenação de Engenharia Química

2 Universidade Estadual de Campinas, Faculdade de Engenharia Química

E-mail para contato: ryk1990@yahoo.com.br; ads.vidotti@ufma.br; guira@feq.unicamp.br; acd.freitas@ufma.br

\begin{abstract}
RESUMO - A reforma autotérmica combina a oxidação parcial com a reforma a vapor em um reator. O processo é dito "autotérmico" quando a reação de reforma endotérmica se processa com a ajuda de uma combustão (oxidação), minimizando os custos energéticos da planta. A reforma autotérmica envolve a reação entre o combustível (metano), o vapor d'água e o oxigênio, este em quantidades menores, apresentando vantagens térmicas sobre os demais processos de reforma. Dentro desse contexto, a reforma autotérmica do metano foi termodinamicamente avaliada com o objetivo de determinar as melhores condições de reação, visando o favorecimento da formação de hidrogênio e/ou gás de síntese. Para isso foi utilizada a metodologia de maximização da entropia, formulada como problema de otimização na forma de programação não-linear, considerando condições de pressão $(\mathrm{P})$ e entalpia $(\mathrm{H})$ constantes. $\mathrm{O}$ modelo foi solucionado com o solver CONOPT3 do software GAMS (General Algebraic Modeling System), versão 23.9.5. A metodologia proposta se mostrou eficiente para a avaliação do comportamento térmico do sistema, verificou-se que o comportamento térmico da reação está diretamente associado com a quantidade de água presente na alimentação, e efeitos de pressão e temperatura que se mostraram significativos também. Produções significativas de hidrogênio e gás de síntese foram encontradas em $1000 \mathrm{~K}, 0 \mathrm{~mol}$ de água na alimentação e 1 bar sendo também essa uma região com comportamento térmico interessante.
\end{abstract}

\section{INTRODUÇÃO}

O consumo energético atual é contínuo e crescente e os combustíveis de origem fóssil são a principal fonte dessa energia, que tem como característica principal o fato de ser nãorenovável. Como alternativa para a demanda de combustíveis de fontes não renováveis tem-se o hidrogênio, que pode ser obtido através de reações de reforma de hidrocarbonetos leves, como o gás natural (Fischer 2001).

Atualmente, dentre os processos de reforma conhecidos, o processo de reforma a vapor do metano, reação entre $\mathrm{CH}_{4}$ e $\mathrm{H}_{2} \mathrm{O}$, é o processo que possui a maior aplicabilidade no setor industrial. Porém, esse processo apresenta alguns gargalos operacionais, tais como elevadas taxas de produção de coque, o que acarreta na desativação rápida do catalisador, além de 
necessitar de um elevado consumo energético, devido a elevada endotermicidade dessa reação (Freitas 2012).

Outros processos de reforma vêm sendo estudados com o objetivo de eliminar as lacunas do processo de reforma a vapor. Tendo em vista o caráter endotérmico da reforma a vapor, a reação de oxidação parcial vem sendo apontada como uma alternativa interessante por suprir essa necessidade, devido basicamente a sua característica exotérmica.

Como alternativa temos o processo de reforma autotérmica, uma junção dos processos de reforma a vapor e reforma oxidativa. Essa reação apresenta as vantagens associadas com ambos os processos (Freitas 2012). As principais reações relacionadas ao processo de reforma autotérmica segundo Aasberg-Petersen et al. (2003), são:

$$
\begin{aligned}
& \mathrm{CH}_{4}+1,5 \mathrm{O}_{2} \leftrightarrow \mathrm{CO}+\mathrm{H}_{2} \mathrm{O} \\
& \mathrm{CH}_{4}+\mathrm{H}_{2} \mathrm{O} \leftrightarrow \mathrm{CO}+3 \mathrm{H}_{2} \\
& \mathrm{H}_{2} \mathrm{O}+\mathrm{CO} \leftrightarrow \mathrm{CO}_{2}+\mathrm{H}_{2}
\end{aligned}
$$

Dentro desse contexto, o objetivo desse trabalho é prever o comportamento térmico da reforma autotérmica do metano utilizando a metodologia de maximização de entropia para sistemas isoentálpicos e isobáricos, bem como avaliar os efeitos das modificações nas condições operacionais, como pressão, temperatura e composição de alimentação, sobre o comportamento térmico da reação.

\section{METODOLOGIA}

Segundo Kondepudi e Prigogine (1998) e Castier (2009) para condições de pressão (P) e entalpia $(\mathrm{H})$ constantes o equilíbrio pode ser determinado ao se obter o máximo da entropia do sistema, essa análise é importante para a determinação de temperaturas de equilíbrio em reatores que possuem reações em condições exotérmicas.

Segundo Freitas (2012) para um problema de maximização da entropia as variáveis consideradas são: número de mols das fases sólida, líquida e gasosa, $n_{i}^{g}, n_{i}^{l}, n_{i}^{s}$, a temperatura do sistema (T) além da própria entropia do sistema (S) bem como todas as quantidades que dependam destes (propriedades físicas dos componentes puros dependentes da temperatura).

A formulação para a maximização da entropia com condições de pressão e entalpia constantes pode ser escrita como:

$$
\max S=\sum_{i=1}^{N C} n_{i}^{g} \bar{s}_{i}^{g}+\sum_{i=1}^{N C} n_{i}^{l} \bar{s}_{i}^{l}+\sum_{i=1}^{N C} n_{i}^{s} \bar{s}_{i}^{s}
$$

Onde foram assumidas às restrições não negatividade do número de mols, equação (5), e conservação de átomos, equação (6) (para sistemas reativos), escritas abaixo. 
- Não negatividade do número de mols:

$$
n_{i}^{k} \geq 0, i=1, \ldots \ldots \ldots . N C, k=1, \ldots \ldots \ldots, N F
$$

Onde NC é o número de componentes presentes no sistema e NF é o número de fases formadas nas condições de pressão e de temperatura admitidas.

Onde $n_{i}^{0}$,é o número de mols total do componente i no sistema.

- Conservação de átomos:

$$
\sum_{i=1}^{N C} a_{m i}\left(\sum_{i=1}^{N C} n_{i}^{k}\right)=\sum_{i=1}^{N C} a_{m i} n_{i}^{0}, m=1, \ldots, N E
$$

Onde $a_{m i}$ é o número de átomos do elemento i no componente i e NE é o número de elementos que possuem o átomo em questão em sua composição.

Uma restrição adicional, tendo a maximização da entropia comparada com a minimização da energia de Gibbs deve ser satisfeita e pode ser escrita da seguinte forma:

$$
\sum_{i=1}^{N C}\left(n_{i}^{g} H_{i}^{g}+n_{i}^{l} H_{i}^{l}+n_{i}^{s l} H_{i}^{s}\right)=\sum_{i=1}^{N C} n_{i}^{0} H_{i}^{0}=H
$$

Onde $H_{i}^{g}+H_{i}^{l}+H_{i}^{s}$ representam a entalpia do componente i nas fases gás, líquida e sólida, respectivamente. H é a entalpia total do sistema e $H_{i}^{0}$ é a entalpia do componente i no estado inicial.

Para a maximização da entropia, a temperatura é variável e assim a capacidade calorífica Cpi, pode ser expressa, de forma genérica como:

$$
C p_{i}=C p_{a i}+C p_{b i} T+C p_{c i} T^{2}+C p_{d i} T^{-2}
$$

Em que $C p_{a i}+C p_{b i}+C p_{c i}+C p_{d i}$ são parâmetros que foram obtidos juntamente com os dados termodinâmicos, para cada substância pura considerada durante as simulações, em Freitas 2012. Para a resolução do problema de otimização, utilizou-se o software GAMS (General Algebraic Modeling System), versão 23.9.5 com o solver CONOPT3. A descrição do software pode ser encontrada em Brooke (1998). Os compostos considerados nas simulações foram $\mathrm{CH}_{4}, \mathrm{O}_{2}$ e $\mathrm{H}_{2} \mathrm{O}$ como reagentes e $\mathrm{CO}, \mathrm{CO}_{2}, \mathrm{H}_{2}, \mathrm{CH}_{4}$ e $\mathrm{H}_{2} \mathrm{O}$ como produtos.

\section{RESULTADOS E DISCUSSÃO}

Neste trabalho foram avaliados os efeitos da variação da razão molar $\mathrm{H}_{2} \mathrm{O} / \mathrm{CH}_{4}$ na alimentação para uma faixa de temperatura de $600 \mathrm{~K}$ a $1100 \mathrm{~K}$ e da pressão de 1 bar a 10 bar com o intuito de verificar a formação de gás de síntese para uma razão molar de água na 
alimentação variada entre $0 \mathrm{~mol}$ e $1 \mathrm{~mol}$. Os efeitos foram estudados sobre a formação dos compostos de interesse da reação e sobre a característica térmica do sistema.

De acordo com a Figura 1 (a), (b) e (c), para 1 mol de água alimentado no sistema é possível notar a produção de hidrogênio em torno de $55 \%$ molar em relação aos demais componentes e que a quantidade de água como produto no sistema está em torno de $20 \%$ molar para todas as pressões. É importante destacar que à temperatura inicial do sistema entre $600 \mathrm{~K}$ e $900 \mathrm{~K}$ proporcionou alterações perceptíveis quanto ao metano restante como produto, verificado com mais clareza à medida que houve o aumento da pressão, mais precisamente a uma pressão de 10 bar, Figura 1(c), em que se tem um percentual molar maior em relação às demais pressões estudadas.

Figura 1 - Percentual da produção para 1,0 mol de água a 1, 5 e 10 bar.

(a)

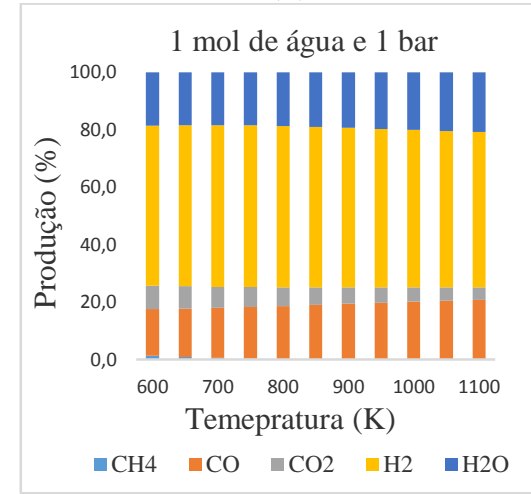

(b)

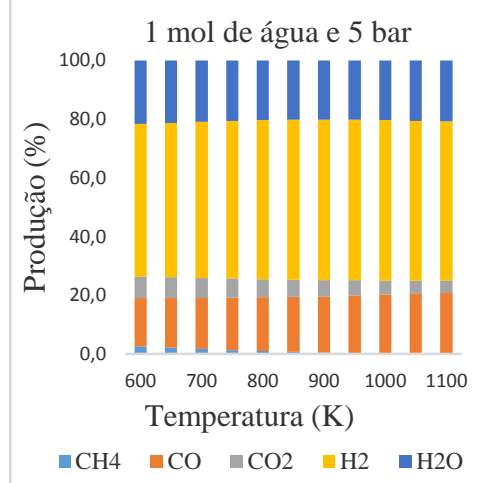

(c)

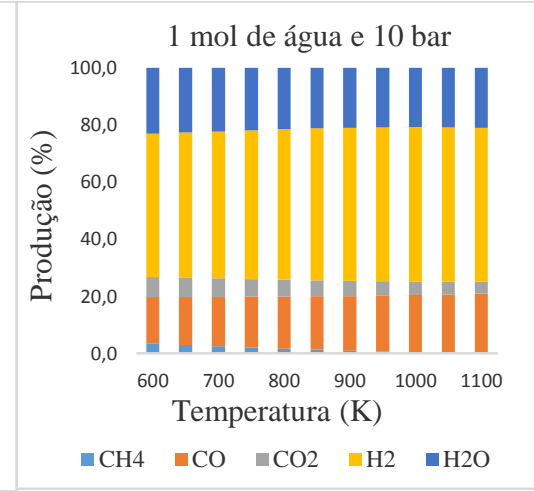

Na figura 2 podemos observar que a produção de hidrogênio para 0,5 mol de água inseridos inicialmente no sistema é em torno de $60 \%$ molar, que é 5\% maior do mostrado na Figura 1, com 55\% molar (em seu máximo na Figura 1 (a)). Quanto à água do sistema, para 0,5 mol inseridos, como mostra a Figura 2, se tem um decréscimo no percentual molar em relação a Figura 1, que teve inicialmente $1 \mathrm{~mol}$ inserido. É interessante notar que a presença do metano não reagido no sistema se mantém com percentual molar semelhante ao visto na Figura 1 e que o efeito da pressão e temperatura se mantem com um comportamento relativamente constante entre essas duas composições de alimentação.

Figura 2 - Percentual da produção para 0,5 mol de água a 1, 5 e 10 bar.

(a)

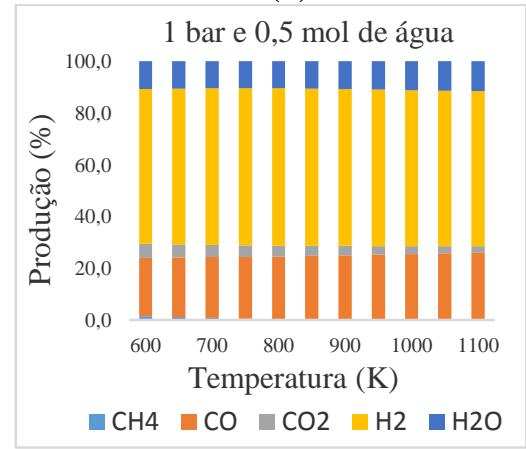

(b)

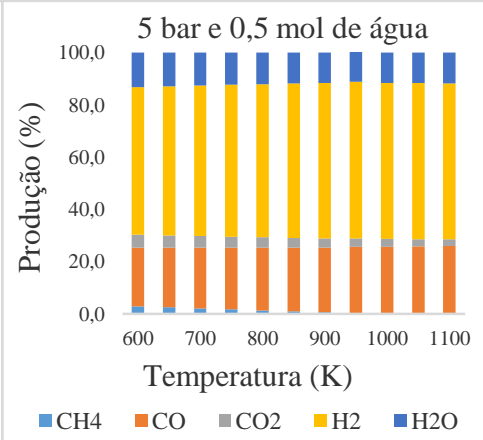

(c)

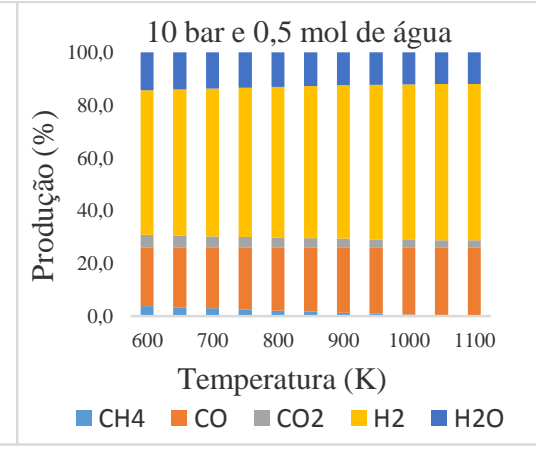


Com a ausência de água no sistema ainda é perceptível a formação desse composto no sistema com uma composição molar em torno de 5\% no máximo, esse comportamento pode ser observado com mais clareza na Figura 3(c) e está diretamente relacionado com o equilíbrio observado na reação de deslocamento gás-água (water-gas-shift). É importante observar que o percentual molar de água produzido no sistema é reduzido à medida que acontece o aumento de temperatura inicial, observado na Figura 3(a), (b) e (c). No que diz respeito à produção de hidrogênio, quando comparamos as Figuras 1, 2 e 3, podemos notar um crescimento no seu percentual para 75\% molar mais ou menos na Figura 3, e que a temperatura bem como a pressão não mostram efeitos significativos para o mesmo nas Figuras 1, 2 e 3 mas sim a quantidade de água inserida no sistema foi o fator de mais importância no crescimento percentual molar do hidrogênio.

Figura 3 - Percentual da produção para 0 mol de água a 1, 5 e 10 bar.

(a)

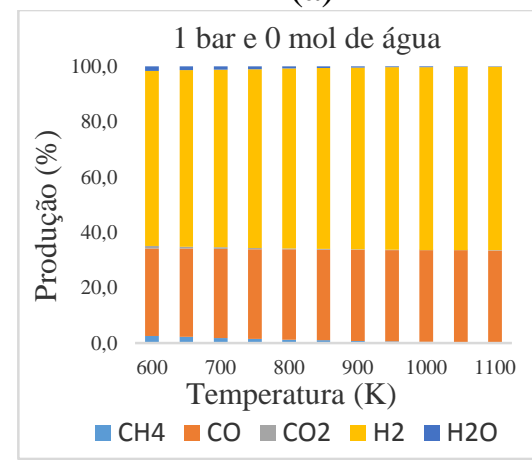

(b)

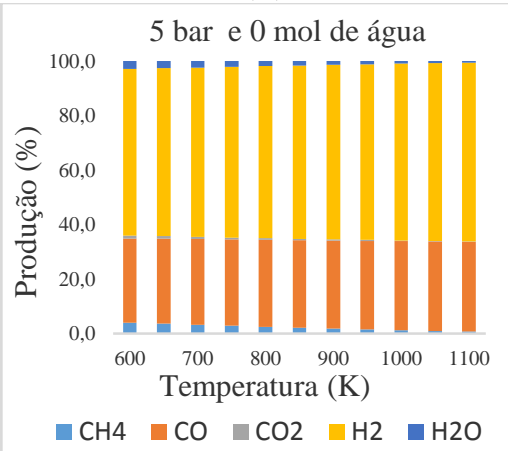

(c)

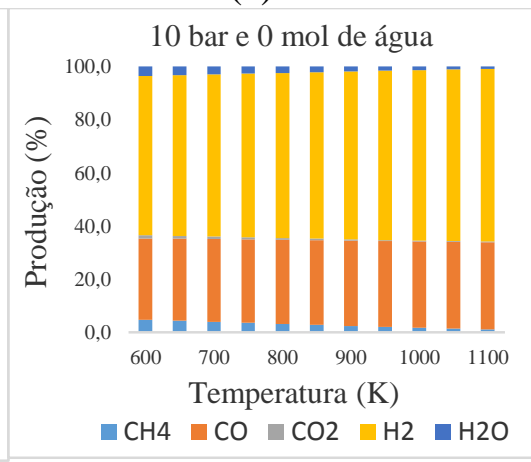

O comportamento térmico da reação, por meio do estudo das temperaturas de equilíbrio observadas para diferentes condições iniciais é apresentado na Figura 4, onde é importante destacar duas características marcantes desse sistema, primeiro o comportamento do mesmo com a variação da pressão, já que a região de maior exotermicidade é atingida a uma pressão de 10 bar, segundo, tem-se que a ausência de água no sistema proporciona, juntamente com a pressão de 10 bar a uma maior liberação de energia, esse comportamento pode ser explicado devido a sobreposição da reação de oxidação parcial, que é altamente exotérmica.

Figura 4 - Temperatura final pela temperatura inicial para $1 \mathrm{~mol}, 0,5 \mathrm{~mol}$ e $0 \mathrm{~mol}$ de oxigênio.

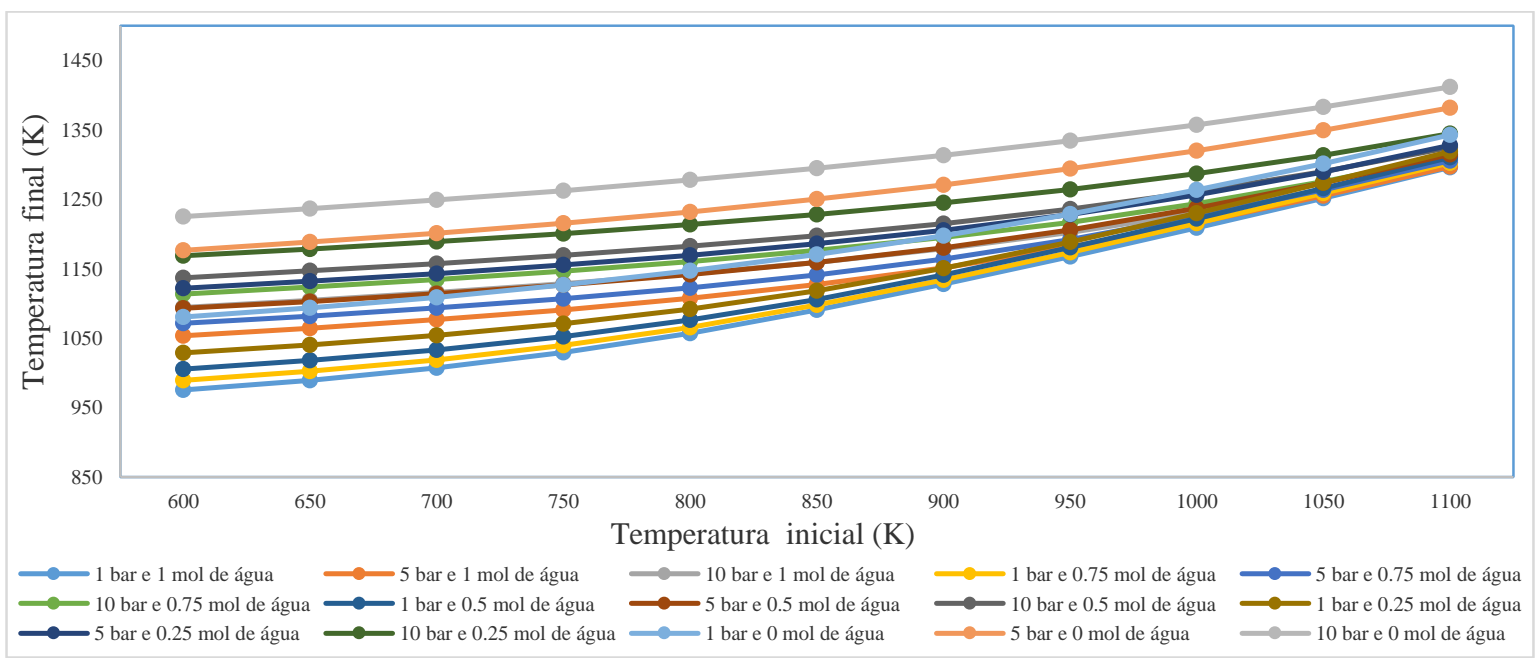




\section{CONCLUSÃO}

Por meio das simulações realizadas utilizando a metodologia de maximização de entropia, foi possível verificar que o processo está diretamente ligado ao ajuste correto da inserção da quantidade de água na alimentação do sistema, e que a reação apresenta um comportamento exotérmico à medida em que essa quantidade de água alimentada é reduzida e aumenta-se a pressão de operação. A uma pressão de 10 bar e não havendo inserção de água no sistema foi alcançada a maior liberação de energia (maior temperatura de equilíbrio) dentre as condições estudadas. É importante destacar que a composição combinada com a pressão apresentam efeitos de deslocamento no desenvolvimento da reação para um caráter endotérmico ou exotérmico, ou para uma maior ou menor produção de hidrogênio, no entanto a temperatura inicial do sistema não se mostrou como um fator de grande relevância quando comparado com os demais fatores estudados. Vale ressaltar que para a combinação de temperatura inicial em torno de $1000 \mathrm{~K}, 1$ bar e 0 mol de água alimentada ao sistema se mostrou uma condição interessante de operação, visando a produção dos compostos de interesse e um comportamento térmico interessante para o sistema.

Portanto, a metodologia utilizada e implementada no software GAMS (General Algebraic Modeling System) 23.9.5, se mostrou eficiente para a avaliação do comportamento térmico do sistema, apresentando bons resultados com baixos tempos computacionais (em torno de 1 segundo).

\section{REFERÊNCIAS}

AASBERG-PETERSEN, K.; CHRISTENSEN, T. S.; NIELSEN, C. S.; DYBKJAER, I. Recent developments in autothermal reforming and pre-reforming for synthesis gas production in GTL applications. Fuel Processing Technology, 83 (2003) 253-261.

BROOKE A., KENDRICK, D., MEEARUS A., RAMAN, R. GAMS - A User's Manual. Washington, DC: In GAMS Development Corp., 1998.

CASTIER, M. Solution of isochoric-isoenergetic flash problem by direct entropy maximization. Fluid Phase Equilibria, 276 (2009) 7-17.

FISCHER, G.; SCHRATTENHOLZER, L. Global bioenergy potentials through 2050. Biomass Bioenergy 2001, 20, 151-159.

FREITAS, A. C. D. Análise Termodinâmica de Processos de Reforma do Metano e da Síntese Fischer- Tropsch: Dissertação de mestrado; Universidade Estadual de Campinas, 2012.

KONDEPUDI, D.; PRIGOGINE, I. Modern thermodynamics: From heat engines to dissipative structures. New York: John Wiley \& Sons Inc, 1998. 